for analgesics may be more important to some patients than the absence of pain. Selected single-shot regional techniques are potentially useful in attaining this outcome.

\section{CONTINUOUS ERECTOR SPINAE PLANE BLOCK FOR PAIN MANAGEMENT IN OPEN RESECTION OF LOCALLY RECURRENT RENAL CELL CARCINOMA - A CASE- REPORT}

${ }^{1}$ AP Pereira* ${ }^{2}{ }^{2}$ Lobo, ${ }^{1} \mathrm{~F}$ Teixeira, ${ }^{1} \mathrm{R}$ Abrunhosa. ${ }^{1}$ Centro Hospitalar de Trás-os-Montes e Alto Douro, Vila Real, Portugal; ${ }^{2}$ Hospital das Forças Armadas - Pólo Porto, Porto, Portugal

\subsection{6/rapm-2021-ESRA.234}

Background and Aims Main issues in postoperative pain management for patients with single kidney include drug dose adjustment and further damage avoidance. Originally described for thoracic analgesia ${ }^{1}$, the erector spinae plane block (ESPB) can provide abdominal somatic and visceral analgesia ${ }^{2,3}$ and circumvent limitations in these patients. We describe the successful use of a continuous ESPB to provide effective perioperative analgesia for renal fossa surgery.

Methods A 79-year-old woman, ASA III, who underwent right nephrectomy due to renal cell carcinoma 4 years before, presented to the operating room for an open resection of renalbed carcinoma recurrence (subhepatic and abdominal wall).

Following general anaesthesia induction, an ultrasoundguided ipsilateral ESPB at T9 level (with $20 \mathrm{ml}$ ropivacaine $0,5 \%$ ) was performed and a catheter was inserted under ultrasound vision. During surgery IV dexamethasone $4 \mathrm{mg}$, fentanyl $0,05 \mathrm{mg}$, paracetamol $1 \mathrm{~g}$ and tramadol $50 \mathrm{mg}$ were administered.

For postoperative pain management we elected IV paracetamol and a ropivacaine $0,2 \%$ ESPB patient-controlled-analgesia device.

Results Patient underwent surgery uneventfully (mean arterial pressure $68-75 \mathrm{mmHg}$ and heart rate $55-68 / \mathrm{min}$ ). She was comfortable after the procedure and safely discharged from anesthesia care. The catheter was used for 2 days and pain was described as 'none' (at rest) or 'mild' (at movement) during vigilance. No rescue opioid was administered. Patient was satisfied with the approach and no postoperative complications were observed.
Conclusions The ESPB appears to be a useful alternative to epidural, paravertebral block or IV strategies for pain management in moderately aggressive retroperitoneal surgeries, with optimal safety profile and extent of sensitive coverage.

\section{EIGHT MG OF EPIDURAL MORPHINE: HOW MUCH SHOULD WE WORRY?}

R Rodrigues Oliveira, A Vasconcelos Pereira*, R Mendes de Carvalho, M Azevedo Coutinho. Hospital Vila Franca de Xira, Vila Franca de Xira, Portugal

\subsection{6/rapm-2021-ESRA.235}

Background and Aims Currently, administering a bolus of 2 to $3 \mathrm{mg}$ of epidural morphine for cesarean section postoperative pain management is widely recommended. Assuming that by using higher doses the increased side effects incidence will outweight any possible benefits in analgesic outcome, there is actually a lack of literature reporting higher dose regimens. We present a case of a patient who accidentally received a dose of $8 \mathrm{mg}$.

Methods A twenty-six-year-old, ASA II female underwent a cesarean section under epidural block due to fetopelvic disproportion. The epidural catheter was previously placed and used for labor analgesia. At the end of the surgery, a bolus of 8 mg of epidural morphine was mistakenly administered instead of $2 \mathrm{mg}$.

The patient was kept in the postoperative care unit for 18 hours and subsequently transferred to the ward. Ondansetron $4 \mathrm{mg} 8 / 8 \mathrm{~h}$ was prescribed for nausea and vomiting prophylaxis.

Results During the $24 \mathrm{~h}$ after the administration, adverse effects such as sedation, nausea, vomiting, pruritus, or urinary retention did not occur. She had no ventilatory depression and was hemodynamically stable throughout the day. Analgesia was optimal without the need of rescue analgesics.

Conclusions Despite the need of adequate monitoring, administering an higher dose of epidural morphine provided optimal analgesia without any adverse effects and allowed for an otherwise normal postoperative period. More studies might be needed in order to determine the actual safety of higher dose epidural morphine and its possible benefits for selected cases. 